\title{
Plasma Biomarkers of AD Emerging as Essential Tools for Drug Development: An EU/US CTAD Task Force Report
}

Randall J. Bateman ${ }^{1}$, Kaj Blennow ${ }^{2}$, Rachelle Doody ${ }^{3}$, Suzanne Hendrix ${ }^{4}$, Simon Lovestone ${ }^{5}$, Stephen Salloway ${ }^{6}$, Rachel Schindler ${ }^{7}$, Michael Weiner ${ }^{8}$, Henrik Zetterberg ${ }^{2,9,10}$, Paul Aisen ${ }^{11}$, Bruno Vellas ${ }^{12}$, and the EU/US CTAD Task Force.

1 - Washington University School of Medicine, St. Louis, MO, USA

2 - Clinical Neurochemistry Laboratory, University of Gothenburg, Sahlgrenska University Hospital, MöIndal, Sweden

3-Genentech/Roche, Basel, Switzerland

4 - Pentara Corporation, Salt Lake City, UT, USA

5 - Janssen Pharmaceuticals, Oxford, UK

6 - The Warren Alpert Medical School of Brown University, Providence RI, USA

7 - Schindler Neuroscience Consulting Group, New York NY, USA

8 - University of California, San Francisco, USA

9 - Department of Neurodegenerative Disease, UCL Institute of Neurology, Queen Square, London, United Kingdom

10 - UK Dementia Research Institute at UCL, London, United Kingdom

11 - Alzheimer's Therapeutic Research Institute (ATRI), Keck School of Medicine, University of Southern California, San Diego, CA, USA

12 - Gerontopole, INSERM U1027, Alzheimer's Disease Research and Clinical Center, Toulouse University Hospital, Toulouse, France

\begin{abstract}
There is an urgent need to develop reliable and sensitive blood-based biomarkers of Alzheimer's disease (AD) that can be used for screening and to increase the efficiency of clinical trials. The European UnionNorth American Clinical Trials in Alzheimer's Disease Task Force (EU/US CTAD Task Force) discussed the current status of blood-based AD biomarker development at its 2018 annual meeting in Barcelona, Spain. Recent improvements in technologies to assess plasma levels of amyloid beta indicate that a single sample of blood could provide an accurate estimate of brain amyloid positivity. Plasma neurofilament light protein appears to provide a good marker of neurodegeneration, although not specific for AD. Plasma tau shows some promising results but weak or no correlation with CSF tau levels, which may reflect rapid clearance of tau in the bloodstream. Blood samples analyzed using -omics and other approaches are also in development and may provide important insight into disease mechanisms as well as biomarker profiles for disease prediction. To advance these technologies, international multistakeholder collaboration is essential.
\end{abstract}




\section{Introduction}

Biomarkers of Alzheimer's disease (AD) are essential tools in drug development to assess the biochemical effects of compounds, accelerate development of compounds designed to counter the disease process, aid in the selection of participants for drug trials, and assess the efficacy of therapies ${ }^{1}$. Clinically, they provide crucial diagnostic information and, when an effective treatment becomes available, they may also be useful as screening or diagnostic tools for early identification and intervention ${ }^{2}$.

In the recently published National Institutes on Aging and Alzheimer's Association (NIA-AA) Research Framework, which defines AD biologically through the use of biomarkers, recognized AD biomarkers included cerebrospinal fluid (CSF) measures of amyloid-beta (A $\beta$ ) and tau, positron emission tomography (PET) assessment of amyloid and tau, and two other imaging measures: anatomic magnetic resonance imaging (MRI) and fluorodeoxyglucose PET (FDG-PET, a measure of brain metabolism) ${ }^{3}$.

Despite their enormous promise in advancing the development of early disease and preventive treatments, the challenges of using CSF and imaging biomarkers limit their use in large diverse populations, in rural areas, and lower- and middle-income countries with limited resources ${ }^{4}$. Many efforts worldwide are meeting this challenge to identify and develop blood-based biomarkers that would enable screening and clinical diagnosis with repeated sampling in clinical trials ${ }^{5}$. Recognizing the urgency of advancing the development of blood-based biomarkers for AD, the European Union-North American Clinical Trials in Alzheimer's Disease Task Force (EU/US CTAD Task Force) addressed this issue at its 2018 meeting in Barcelona, Spain. The Task Force provided a forum for investigators from the pharmaceutical and diagnostics industries to join researchers from academia and regulatory agencies in efforts to build consensus on the path forward in developing and bringing to market blood-based biomarkers tests.

Many challenges have been encountered in efforts to identify reliable, sensitive, and specific biomarkers of $A D$ in plasma or serum. The close and continuous contact of the brain with the CSF results in relatively high levels of specific molecules associated with brain disease, while much lower amounts exist in the bloodstream ${ }^{5}$. Further complicating the measurement of plasma-based AD biomarkers are high levels of other proteins from peripheral organs in the blood and the presence of proteases that may degrade brain proteins. 
Nonetheless, in recent years there have been dramatic improvements in highly sensitive and specific immunoassays and mass spectrometry-based assays used to assess plasma levels of molecules that could serve as biomarkers of AD and other types of neurodegeneration ${ }^{6}$. These advances have increased optimism in the field regarding the use of blood-based biomarker "profiles" for diagnosis, prognosis, and disease progression monitoring ${ }^{7}$. Blood-based biomarkers are also seen as an essential part of efforts to develop precision medicine approaches for $A D^{8,9}$.

\section{Plasma amyloid beta}

Longitudinal studies in individuals with autosomal dominant forms of AD have shown that CSF levels of $A \beta 42$ decline 25 years before expected symptom onset; and that amyloid plaques are detectable by PET imaging 15 years before expected symptom onset ${ }^{10}$. Early attempts to measure $A \beta$ peptides in plasma indicated that these tests had limited value as tools for diagnosis or prognosis ${ }^{4}$, but these studies were based on comparing plasma $A \beta$ in clinically diagnosed $A D$ patients and cognitively unimpaired elderly, which, given the uncertainty of $A D$ diagnosis and overlap in pathology, limits the chance to identify minor changes in biomarker levels, as compared with using brain amyloid positivity as the reference standard. High variability was attributed, in part, to a lack of standardized protocols and methods. In addition, plasma $A \beta$ originates not only in the brain but also in other organs and tissues ${ }^{11}$.

Recent improvements in the technologies used to assess plasma levels of $A \beta$ have shown more promising results. For example, investigators at Washington University have demonstrated that the ratio of plasma $A \beta 42 / 40$ provides a sensitive and reliable measure of amyloid status that predicts future conversion to positive amyloid PET independent of the time of day and correlates with CSF AB42/40 ${ }^{12}$. Other studies in European memory clinics ${ }^{13}$, the Swedish Bio/FINDER cross-sectional and ESTHER longitudinal cohorts ${ }^{14}$, the Australian Imaging, Biomarker and Lifestyle Flagship Study (AIBL) cohort ${ }^{15}$, and the National Center for Geriatrics and Gerontology (NCGG) Hospital in Japan ${ }^{16,17}$ have also shown good correlations with amyloid PET.

These results indicate that plasma $A \beta 42 / 40$, combined with age and $A P O E$ status performs as well as CSF or PET and can be used with a high degree of sensitivity and specificity ( $>90 \%)$ to detect AD amyloid plaques in individuals before symptom onset. For clinical use, a single sample of blood could provide a highly accurate estimate of who is amyloid positive and thus support the diagnosis of AD[REF 12] ${ }^{17}$; while in clinical trials, a blood $A \beta 42 / 40$ test could be used as a prescreening tool to identify who is has or is at risk for $A D$ and facilitate efficient and cost-efficient recruitment of participants, thus accelerating trials, lowering costs, and speeding drug discovery[REF 12] ${ }^{17}$. For example, it is estimated 
that more than $50 \%$ of amyloid PET scans could be avoided with blood-based screening for A $\beta$ pathology in the brain. [REF Bateman CTAD presentation]

\section{Plasma tau}

In CSF, total tau (T-tau) and phosphorylated tau (P-tau) have been well validated as biomarkers of $A D^{18}$. In the $A / T / N$ classification system, P-tau represents biomarker of tau pathology and CSF T-tau also represents a biomarker of neuronal injury or neurodegeneration ${ }^{19}$, although recent data on the kinetics of tau suggests that in $A D, C S F$ tau reflects increased neuronal secretion of tau in response to $A \beta$ pathology rather than neurodegeneration ${ }^{20}$.

Several studies have reported that T-tau levels are also elevated in the plasma of people with $A D$, although there is substantial overlap between diagnostic groups (cognitively normal, $M C l, A D){ }^{21,} 22$. T-tau in CSF and plasma is elevated in other disorders involving substantial brain injury, such as Creutzfeldt-Jacob disease (CJD) ${ }^{23,24}$, stroke ${ }^{25}$, cardiac arrest ${ }^{26}$, and traumatic brain injury ${ }^{27}$. P-tau181 levels are also elevated in AD dementia and show better associations with both $A \beta$ and tau PET, suggesting greater specificity for AD pathology ${ }^{28}$.

In regards to P-tau, a semi-sensitive assay for tau phosphorylated at threonine 181 (similar to the most employed CSF test) with electrochemiluminescence detection has been developed (Mielke MM, Hagen CE, Xu J, Chai X, Vemuri P, Lowe VJ, Airey DC, Knopman DS, Roberts RO, Machulda MM, Jack CR, Jr., Petersen RC, Dage JL. Plasma phospho-tau181 increases with Alzheimer's disease clinical severity and is associated with tau- and amyloid-positron emission tomography. Alzheimers Dement, 2018; 14 : 989-97). Using this assay, plasma P-tau concentration was higher in AD dementia patients than controls. Plasma P-tau concentration was associated with both $A \beta$ and tau PET, which is a promising result in need of replication.

The expression of tau is brain-enriched, but tau is also detectable at both mRNA and protein level in salivary glands and kidney (http://www.proteinatlas.org/ENSG00000186868-MAPT/tissue). This is an important potential confounder that may help explain the weak correlation of plasma with CSF tau. The weak correlation may also reflect rapid clearance of tau in the bloodstream ${ }^{29,} 30$.

\section{Neurofilament light (NFL)}

Neurofilament light chain (NFL) is an intraneuronal protein and a component of the axonal cytoskeleton, thus its presence in the CSF indicates neuronal damage or degeneration ${ }^{31}$. In AD, CSF NFL 
concentrations increase in early stages of disease and increase over time as cognition declines and atrophy and white matter changes in the brain increase ${ }^{32}$.

In a recent study comparing three analytical platforms for assessing NFL in serum, the singlemolecule array (Simoa) method is emerging as more sensitive than conventional enzyme-linked immunosorbent assay (ELISA) or electroluminescence (ECL) ${ }^{33}$. A large study in the ADNI population using the Simoa assay showed that plasma NFL correlates with CSF NFL as an indicator of neurodegeneration across the $A D$ continuum, has diagnostic accuracy for AD dementia similar to that of CSF biomarkers, and is associated with cognitive decline and neuroimaging biomarkers of $\operatorname{AD}^{34,35}$. Similarly, in a study conducted in Germany using the Simoa method, plasma NFL concentrations were significantly higher in people with $\mathrm{MCl}$ and $\mathrm{AD}$ dementia compared to normal controls even after correcting for age ${ }^{36}$. Plasma NFL concentrations were also inversely correlated with Mini Mental Status Examination (MMSE) scores, which suggests that unlike other CSF biomarkers of AD, increased NFL may indicate ongoing neurodegeneration and functional decline ${ }^{36}$. These studies suggest that NFL may have potential for prognosis and monitoring of disease progression. A small study in patients with familial $A D$ suggested that plasma NFL increases about 5 years prior to estimate onset, suggesting its utility as a screening tool ${ }^{37}$, and a larger study in the Dominantly Inherited Alzheimer Network (DIAN) demonstrated serum NFL correlates with neurodegeneration and clinical decline and longitudinal change identifies mutations carriers 16 years before symptom onset. [REF Nat Med 2019]

However, NFL is not specific for AD, but a general neurodegeneration biomarker, for review see (REF). Knowledge about the usefulness of NFL as a biomarker for neurodegeneration emerged in large part from studies in multiple sclerosis, and it has also been used to assess CNS injury in HIV infection, frontotemporal dementia, amyotrophic lateral sclerosis (ALS), CJD, Parkinson's disease (PD) and other CNS disorders ${ }^{34,37,38}$. One study in people with HIV infection suggested that plasma NFL may be useful to monitor downstream drug effects on the intensity of neurodegeneration (ref:

https://www.ncbi.nlm.nih.gov/pubmed/30105502). In patients with CJD, elevations of both tau and NFL in serum at baseline predict steeper increases over time ${ }^{24}$. Studies in patients with multiple sclerosis also suggest a role for NFL as an indicator of treatment effectiveness ${ }^{39,40}$.

Differences in the preanalytic handling of serum samples was shown to significantly affect the measurement of NFL, pointing to the importance of standardized protocols for sample collection, storage, and transport ${ }^{36}$.

\section{Omics and other approaches}


Blood samples are also useful for obtaining high-dimensional biomarker profiles using a combination of omics approaches, including genomics, transcriptomics, metabolomics, lipidomics, and proteomics. Advances in mass spectrometry have even enabled the molecular characterization of biological processes from single cells ${ }^{41}$. These approaches enable the discovery of unknown unknowns and may also provide insight into molecular mechanisms that underlie diseases such as AD.

Different approaches may be used to harness the power of these technologies for omics studies

${ }^{6}$. However, the choice of method may have substantial implications on what is found, and thus interpretation of omics studies must take into account the approach used. For example, Hye and colleagues used mass spectrometry and 2-D gel electrophoresis in a case-control approach comparing the plasma proteomes from elderly people with $A D$ and normal elders ${ }^{42}$. They found an elevation in complement factor $\mathrm{H}$, and this finding was subsequently replicated in multiple studies. Using the same technology with an endophenotype approach in people with $A D$, where discovery was predicated on either hippocampal atrophy or speed of progression, these same investigators showed that elevations of plasma clusterin - an amyloid chaperone -- was associated with both endophenotypes ${ }^{43}$. This finding has also been widely replicated.

Now, the European Medical Information Framework - Multimodal Biomarker Discovery (EMIF$\mathrm{MBD}$ ) project is using an endophenotype approach to identify biomarkers (including plasma biomarkers) of pre-dementia AD. The endophenotypes selected for this multicenter study include amyloid positivity assessed by PET or CSF, MCl conversion to $\mathrm{AD}$, and the rate of cognitive decline. First, they analyzed results from 10 years of studies using multiple omics approaches, which allowed them to identify 7 proteins predictive of amyloid positivity. Next, they used an aptamer capture array provided by SomaLogic to measure 4,600 plasma proteins simultaneously. A machine learning approach revealed 46 features (44 proteins plus ApoE status and age) that predicted amyloid positivity with an area under the curve ( $A \cup C$ ) Of .78, which indicates fair accuracy. Even in people with no signs of $A D$, the 46 features predicted preclinical AD with an AUC of .68, which is statistically significant. While this is nowhere near the accuracy of a well-targeted protein study such as CSF A $\beta$, tau, or plasma NFL, with additional refinement, it could be useful for screening large populations to identify potential candidates for clinical studies targeting preclinical AD.

\section{Conclusions}

Studies completed in the last few years have produced substantial data supporting the further development and potential uses of blood-based biomarkers. Multiple groups have shown that plasma 
$A \beta$ studies can be highly precise in diagnosing amyloidosis, suggesting that a blood-based test of $A \beta$ may enable screening of large populations to identify who is at risk for AD and start intervention before memory loss and brain damage. Plasma NFL has also been shown to be indicative of neurodegeneration in many populations, including in the DIAN population to measure progression, onset, and decline; as well as in sporadic AD. The Task Force concluded that with commercialization efforts underway for both of these tests, they may be validated and ready for clinical use relatively soon.

Current data are less supportive of the use of plasma tau as a useful biomarker for $A D$, at least using the current assay formats, which are based on N-terminal and mid-domain tau antibodies, although this remains a very active area of investigation. Proteomics appear to be useful primarily to search and find targets but may not be useful as inclusion criteria or outcome measures. Many other biomarkers are also being investigated, but thus far none has risen to the standards set by PET scans and CSF measures.

To advance development of plasma-based biomarkers for drug development and clinical use, the Task Force concluded that global standardization and harmonization of preanalytical and analytical protocols will be necessary, which will require international multi-stakeholder collaboration ${ }^{7,44}$. In addition, large scale, longitudinal validation studies will be needed, and the usefulness of plasma $A \beta$ markers to monitor disease progression in clinical trials will need to be determined ${ }^{17}$. To help facilitate such studies, ADNI has huge numbers of coded and blinded plasma and CSF samples available upon request.

1. Hampel H, Frank R, Broich $\mathrm{K}$, et al. Biomarkers for Alzheimer's disease: academic, industry and regulatory perspectives. Nat Rev Drug Discov 2010;9:560-574.

2. Hampel H, Broich K, Hoessler Y, Pantel J. Biological markers for early detection and pharmacological treatment of Alzheimer's disease. Dialogues Clin Neurosci 2009;11:141-157. 3. Jack CR, Jr., Bennett DA, Blennow K, et al. NIA-AA Research Framework: Toward a biological definition of Alzheimer's disease. Alzheimers Dement 2018;14:535-562.

4. Henriksen K, O'Bryant SE, Hampel H, et al. The future of blood-based biomarkers for Alzheimer's disease. Alzheimers Dement 2014;10:115-131.

5. Blennow K, Zetterberg H. The Past and the Future of Alzheimer's Disease Fluid Biomarkers. J Alzheimers Dis 2018;62:1125-1140.

6. Shi L, Baird AL, Westwood S, et al. A Decade of Blood Biomarkers for Alzheimer's Disease Research: An Evolving Field, Improving Study Designs, and the Challenge of Replication. J Alzheimers Dis 2018;62:1181-1198.

7. Hampel H, Vergallo A, Bonuccelli U, Lista S. Editorial: Turning Point towards Blood BiomarkerGuided Targeted Therapy for Precision Medicine in Alzheimer's disease. J Prev Alzheimers Dis 2018;5:160-164.

8. Hampel H, Toschi N, Babiloni C, et al. Revolution of Alzheimer Precision Neurology. Passageway of Systems Biology and Neurophysiology. J Alzheimers Dis 2018;64:S47-S105. 
9. Hampel H, Vergallo A, Aguilar LF, et al. Precision pharmacology for Alzheimer's disease. Pharmacol Res 2018;130:331-365.

10. Bateman RJ, Xiong C, Benzinger TL, et al. Clinical and biomarker changes in dominantly inherited Alzheimer's disease. N Engl J Med 2012;367:795-804.

11. Toledo JB, Shaw LM, Trojanowski JQ. Plasma amyloid beta measurements - a desired but elusive Alzheimer's disease biomarker. Alzheimers Res Ther 2013;5:8.

12. Ovod V, Ramsey KN, Mawuenyega KG, et al. Amyloid beta concentrations and stable isotope labeling kinetics of human plasma specific to central nervous system amyloidosis. Alzheimers Dement 2017;13:841-849.

13. Perez-Grijalba V, Romero J, Pesini P, et al. Plasma Abeta42/40 Ratio Detects Early Stages of Alzheimer's Disease and Correlates with CSF and Neuroimaging Biomarkers in the AB255 Study. J Prev Alzheimers Dis 2019;6:34-41.

14. Nabers A, Perna L, Lange J, et al. Amyloid blood biomarker detects Alzheimer's disease. EMBO Mol Med 2018;10.

15. Fandos N, Perez-Grijalba V, Pesini P, et al. Plasma amyloid beta $42 / 40$ ratios as biomarkers for amyloid beta cerebral deposition in cognitively normal individuals. Alzheimers Dement (Amst) 2017;8:179-187.

16. Kaneko N, Nakamura A, Washimi Y, et al. Novel plasma biomarker surrogating cerebral amyloid deposition. Proc Jpn Acad Ser B Phys Biol Sci 2014;90:353-364.

17. Nakamura A, Kaneko N, Villemagne VL, et al. High performance plasma amyloid-beta biomarkers for Alzheimer's disease. Nature 2018;554:249-254.

18. Olsson B, Lautner R, Andreasson U, et al. CSF and blood biomarkers for the diagnosis of Alzheimer's disease: a systematic review and meta-analysis. Lancet Neurol 2016;15:673-684.

19. Jack CR, Jr., Bennett DA, Blennow K, et al. A/T/N: An unbiased descriptive classification scheme for Alzheimer disease biomarkers. Neurology 2016;87:539-547.

20. Sato C, Barthelemy NR, Mawuenyega KG, et al. Tau Kinetics in Neurons and the Human Central Nervous System. Neuron 2018;97:1284-1298.

21. Mielke MM, Hagen CE, Wennberg AMV, et al. Association of Plasma Total Tau Level With Cognitive Decline and Risk of Mild Cognitive Impairment or Dementia in the Mayo Clinic Study on Aging. JAMA Neurol 2017;74:1073-1080.

22. Zetterberg $\mathrm{H}$, Wilson $\mathrm{D}$, Andreasson $\mathrm{U}$, et al. Plasma tau levels in Alzheimer's disease. Alzheimers Res Ther 2013;5:9.

23. Skillback T, Rosen C, Asztely F, Mattsson N, Blennow K, Zetterberg H. Diagnostic performance of cerebrospinal fluid total tau and phosphorylated tau in Creutzfeldt-Jakob disease: results from the Swedish Mortality Registry. JAMA Neurol 2014;71:476-483.

24. Thompson AGB, Luk C, Heslegrave AJ, et al. Neurofilament light chain and tau concentrations are markedly increased in the serum of patients with sporadic Creutzfeldt-Jakob disease, and tau correlates with rate of disease progression. J Neurol Neurosurg Psychiatry 2018;89:955-961.

25. Hesse $\mathrm{C}$, Rosengren $\mathrm{L}$, Andreasen $\mathrm{N}$, et al. Transient increase in total tau but not phospho-tau in human cerebrospinal fluid after acute stroke. Neurosci Lett 2001;297:187-190.

26. Randall J, Mortberg E, Provuncher GK, et al. Tau proteins in serum predict neurological outcome after hypoxic brain injury from cardiac arrest: results of a pilot study. Resuscitation 2013;84:351-356.

27. Ost M, Nylen K, Csajbok L, et al. Initial CSF total tau correlates with 1-year outcome in patients with traumatic brain injury. Neurology 2006;67:1600-1604.

28. Mielke MM, Hagen CE, XU J, et al. Plasma phospho-tau181 increases with Alzheimer's disease clinical severity and is associated with tau- and amyloid-positron emission tomography. Alzheimers Dement 2018;14:989-997. 
29. Evered L, Silbert B, Scott DA, Zetterberg H, Blennow K. Association of Changes in Plasma Neurofilament Light and Tau Levels With Anesthesia and Surgery: Results From the CAPACITY and ARCADIAN Studies. JAMA Neurol 2018;75:542-547.

30. Zetterberg H. Review: Tau in biofluids - relation to pathology, imaging and clinical features. Neuropathol Appl Neurobiol 2017;43:194-199.

31. Teunissen CE, Khalil M. Neurofilaments as biomarkers in multiple sclerosis. Mult Scler 2012;18:552-556.

32. Zetterberg H, Skillback T, Mattsson N, et al. Association of Cerebrospinal Fluid Neurofilament Light Concentration With Alzheimer Disease Progression. JAMA Neurol 2016;73:60-67.

33. Kuhle J, Barro C, Andreasson U, et al. Comparison of three analytical platforms for quantification of the neurofilament light chain in blood samples: ELISA, electrochemiluminescence immunoassay and Simoa. Clin Chem Lab Med 2016;54:1655-1661.

34. Gisslen M, Price RW, Andreasson U, et al. Plasma Concentration of the Neurofilament Light Protein (NFL) is a Biomarker of CNS Injury in HIV Infection: A Cross-Sectional Study. EBioMedicine 2016;3:135-140.

35. Mattsson N, Andreasson U, Zetterberg H, Blennow K, Alzheimer's Disease Neuroimaging I. Association of Plasma Neurofilament Light With Neurodegeneration in Patients With Alzheimer Disease. JAMA Neurol 2017;74:557-566.

36. Lewczuk P, Ermann N, Andreasson U, et al. Plasma neurofilament light as a potential biomarker of neurodegeneration in Alzheimer's disease. Alzheimers Res Ther 2018;10:71.

37. Weston PSJ, Poole T, Ryan NS, et al. Serum neurofilament light in familial Alzheimer disease: A marker of early neurodegeneration. Neurology 2017;89:2167-2175.

38. Hansson O, Janelidze S, Hall S, et al. Blood-based NfL: A biomarker for differential diagnosis of parkinsonian disorder. Neurology 2017;88:930-937.

39. Novakova L, Zetterberg $\mathrm{H}$, Sundstrom $\mathrm{P}$, et al. Monitoring disease activity in multiple sclerosis using serum neurofilament light protein. Neurology 2017;89:2230-2237.

40. Piehl F, Kockum I, Khademi M, et al. Plasma neurofilament light chain levels in patients with MS switching from injectable therapies to fingolimod. Mult Scler 2018;24:1046-1054.

41. Couvillion SP, Zhu Y, Nagy G, et al. New mass spectrometry technologies contributing towards comprehensive and high throughput omics analyses of single cells. Analyst 2018.

42. Hye A, Lynham S, Thambisetty M, et al. Proteome-based plasma biomarkers for Alzheimer's disease. Brain 2006;129:3042-3050.

43. Thambisetty $M$, Simmons A, Velayudhan $L$, et al. Association of plasma clusterin concentration with severity, pathology, and progression in Alzheimer disease. Arch Gen Psychiatry 2010;67:739-748.

44. Nakamura A. Editorial: Plasma Biomarker for Alzheimer's Disease: Are We Ready Now for Clinical Practice and Drug Trials? J Prev Alzheimers Dis 2018;5:158-159. 